\title{
Knowledge and Attitude of Medical Residents Towards Cancer Clinical Trials in Jordan
}

This article was published in the following Dove Press journal:

International Journal of General Medicine

\author{
Ahmad Al-Azayzih (1D ${ }^{1,2}$ \\ Karem H Alzoubi ${ }^{2}$ \\ 'Department of Pharmacology and \\ Therapeutics, College of Medicine and \\ Health Sciences, United Arab Emirates \\ University, Al Ain, United Arab Emirates; \\ ${ }^{2}$ Department of Clinical Pharmacy, \\ Faculty of Pharmacy, Jordan University of \\ Science and Technology, Irbid 221 I0, \\ Jordan
}

Background: Clinical trials are an important tool to test the efficacy of new treatment modalities for cancer patients. Physicians, including medical residents, should play a major role in carrying out clinical trials to generate a strong body of evidence to determine the best available treatment for their patients. Carrying out clinical trials demands adequate understanding of the research phases and requirements including ethical standards as well as presenting positive attitudes toward the clinical research. Hence, evaluating the knowledge and attitudes of medical residents toward running clinical trials is essential to assess their preparedness and willingness to participate in future studies.

Methods: This study was a questionnaire-based observational study. It involved medical residents from various specialties who served cancer patients admitted at King Abdullah University Hospital during the period from June 1 to August 15, 2017.

Results: A total number of 83 respondents completed the questionnaire. Of them, $56.7 \%$ and $53.0 \%$ of the respondents reported either current or previous participation in clinical trials research, respectively. Only 10 residents $(12.0 \%)$ had previous participation in clinical research where a new investigational cancer treatment was tested. While, $91.6 \%$ of respondents believed that physicians should be involved in running clinical cancer research, only $25.3 \%$ had previous experience in writing a cancer clinical trial protocol and $28.9 \%$ wrote a scientific manuscript on cancer clinical trials for publication. Moreover, $67.5 \%$ of residents knew when informed consent should be obtained and $62.7 \%$ were aware of the clinical equipoise concept in clinical trials.

Conclusion: Much remains to be done to improve knowledge and attitudes of medical residents toward cancer clinical trials and the main ethical principles that should be followed to assure having an ideal research environment, which will pave the way for the generation of high quality clinical cancer research and reliable evidence-based clinical practice for cancer management.

Keywords: clinical trials, cancer, Jordan, oncology, medical residents

\section{Introduction}

Cancer clinical trials are usually done by physicians to test new ways to treat cancer or find a new cancer prevention method, or to test a new diagnostic or screening test for cancer. ${ }^{1}$ There are many regulations and standards that were set up to regulate clinical trials worldwide. Thus, it is very important for investigators who are generally involved with this type of research to understand and apply those standards when they run clinical trials among cancer patients. ${ }^{2}$

A number of oncological diseases have poor prognosis and there is an eminent need for new-targeted therapies that give the oncologist improved methods of cancer treatment with higher efficacy and minimal unwanted adverse drug
Correspondence: Ahmad Al-Azayzih Department of Pharmacology and Therapeutics, College of Medicine and Health Sciences, United Arab Emirates University, Al Ain, United Arab Emirates Email aazayzih@uaeu.ac.ae 
reactions. ${ }^{3,4}$ Clinical research in oncology presents several ethical issues, as efforts are ongoing to better understand mechanisms of cancer progression and metastasis, hence new therapeutic drug targets are available for testing in clinical trials as potential anticancer agents. ${ }^{5}$ One of the major ethical issues encountered in clinical trials that deal with new targeted anticancer therapy is that it is difficult to identify patients that would benefit from the new treatment before the beginning of the trials. This could expose the patients to unwanted health consequences resulting from the new agents without obtaining any recognizable anticancer benefits. ${ }^{5,6}$

In 1998, the national institute of health (NIH) in United Sates implemented a number of new policies to encourage carrying out clinical research by young health-care practitioners. ${ }^{7}$ Nowadays, integration of clinical research concepts into training curricula is mandatory by many of the medical residency programs as well as the accreditation council for graduate medical education (ACGME) of the USA and other medical councils worldwide. ${ }^{8,10}$ Clinical research during the residency period could involve a wide range of forms including case reports, cross-sectional studies, writing a meta-analysis or systemic review, or involvement in randomized controlled clinical trials. During research training, medical residents should be able to comprehend successfully all the necessary phases of research involving the preparatory phase, investigation phase and synthesis task. ${ }^{9}$ Finding a research topic and mentors, developing the skills to design a study, and completion of institutional review board requirements and obtaining ethical approval are among the essential steps to be followed by clinical researchers before moving on with further data collection, statistical analysis and writing conference abstracts or full manuscripts. ${ }^{9}$

Previous studies surveyed medical residents about their research experience during their residency training. The majority of the residents showed positive attitudes toward their research projects. ${ }^{11,12}$ In fact, research part experience received a high rating evaluation by residents compared to other components of residency curriculum. ${ }^{12}$ Another study revealed that family medicine residents who were involved in research projects showed higher recognition toward practicing the evidence-based medicine and its role in improvement in medical practice. ${ }^{13}$

In Jordan, several hospitals and research centers have been authorized by the Jordan Food and Drug Administration (JFDA) to run various phases of clinical trials ranging from bioequivalence studies to performing clinical trials on patients. ${ }^{14}$ The Pharmaceutical Research Center (PRC) located at the King Abdullah University Hospital (KAUH), Jordan University of Science and Technology is among the leading research centers in Jordan. Since the establishment of the PRC in 2004, more than 45 clinical trials (phase I to phase IV) and 300 bioanalytical studies were carried out by investigators at KAUH and other affiliated institutions such as national pharmaceutical companies and other academic institutions. ${ }^{15}$ Medical residents at this hospital and other teaching and research hospitals are expected to demonstrate adequate understanding of clinical research requirements and apply their research outcomes toward improvement of patients' care.

In the current study, both knowledge and attitude of medical residents toward cancer clinical trials and main barriers and ethical issues that lead to an inadequate number of oncological clinical trials involving cancer therapies in Jordan were investigated. Our future aim is providing recommendations to improve the knowledge of health care teams that are involved in or will be involved in clinical trials regarding related ethical challenges.

\section{Ethical Approval}

Approval of the study protocol was granted by the Institutional Review Boards of Jordan University of Science and Technology and the KAUH.

\section{Methods \\ Study Design}

This study was a questionnaire-based observational study.

\section{Inclusion Criteria}

Medical residents from various medical specialties who served oncology patients at internal medicine, adult oncology, pediatric oncology, and surgical oncology units at KAUH in Jordan were included in the study. A total number of 83 residents were interviewed and asked to complete the questionnaire during the period from (June 1 to August 15, 2017).

\section{Questionnaire}

A literature review was performed to determine the core points utilized to assess attitudes and knowledge of medical residents toward clinical trials. ${ }^{16,17}$ The questionnaire items were tailored to suit the purposed study objectives focusing on cancer-related clinical trials. An informed consent statement was included at the beginning of the questionnaire with a brief description of the study purpose 
and the questionnaire participants provided their informed consent for their participation. The questionnaire consisted of three major domains including medical residents individualized characteristics such as gender, age, and current medical rotation specialty. The second domain of the questionnaire inquired about medical residents' attitudes toward cancer clinical trials research. Six close-ended questions with either "yes" or "no" answers were used to assess the attitudes domain. Medical residents were asked about their previous and current participation in cancer clinical trials, and whether their previous participation in clinical trials involved using a new investigational drug for cancer patients. In addition, residents were asked if they had received previous training on how to carry out clinical research. Finally, medical residents' attitudes toward prospective participation in running clinical trials were assessed and if it was important for them to be involved in clinical research. The third domain of the questionnaire inquired about medical residents' knowledge toward clinical trials related to oncology. Seven close-ended type questions of which five were "yes" or "no" answers. Medical residents were asked if they had previously written or at least participated in writing a clinical protocol, and if they had ever submitted a research manuscript on clinical cancer research during their residency training. The knowledge domain involved assessment of residents' knowledge about the international ethical standards regulating the clinical trials in general, and more specifically, cancer clinical trials. Moreover, they were asked about their knowledge of the Declaration of Helsinki as well as clinical equipoise concept in clinical trials. Finally, the questionnaire included one open-ended question to explore the resident's opinions about the main ethical issues that might face the administration of clinical trials, especially those connected with cancer therapies in Jordan.

\section{Data Analysis}

The Statistical data analyses of averages and standard deviations, frequencies, and percentages were performed using SPSS program version 17.0 (SPSS Inc., Chicago, IL, USA).

\section{Results}

A total number of 83 medical residents from different medical fields and specialties responded and completed the study questionnaire. The majority of medical residents were males $(\mathrm{N}=68,81.9 \%)$. Their average age $\pm \mathrm{SD}$ was $30 \pm 3.6$ years. (Table 1). Among respondents, 47 reported that they have current participation in oncological clinical trials and 44
Table I Medical Residents Individualized Characteristics

\begin{tabular}{|l|l|l|}
\hline Variable & Category & N (\%) \\
\hline Gender & Male & $68(81.9)$ \\
& Female & $15(18.1)$ \\
& Both & $83(100)$ \\
\hline Medical specialty & Medical oncology & $18(21.7)$ \\
& Surgical oncology & $39(47.0)$ \\
& Pediatric oncology & $4(4.8)$ \\
& Internal medicine & $22(26.5)$ \\
\hline
\end{tabular}

Note: Average age in years $\pm S D=30 \pm 3.6$.

reported a previous participation in cancer research (Table 2). Only 10 of them reported previous or current participation in clinical trials that involved using a new therapeutic drug for cancer patients (Table 2). Most of the residents showed a positive attitude toward future participation in cancer clinical trials and almost all of them believed that physicians should be involved in clinical research (Table 2). A total number of 21 of the respondents $(25.3 \%)$ reported that they have previously helped in writing a research protocol under the supervision of their mentors and only 24 of them reported they have submitted a research manuscript submitted to international journals (Table 3). Only one of the residents reported a full understating of the Declaration of Helsinki, which is a fundamental document on human research and clinical trials ethics (Table 3).

Table 2 Attitude of Medical Residents Toward Cancer Clinical Trials

\begin{tabular}{|l|l|}
\hline Item & N (\%) \\
\hline $\begin{array}{l}\text { Do you have current participation in cancer clinical trials? } \\
\text { Yes }\end{array}$ & $47(56.7)$ \\
\hline $\begin{array}{l}\text { Do you have previous participation in cancer clinical } \\
\text { trials? } \\
\text { Yes }\end{array}$ & 44 (53.0) \\
\hline $\begin{array}{l}\text { Does your previous or current clinical research involve } \\
\text { using new } \\
\text { investigation drug for cancer patients? } \\
\text { (Yes) }\end{array}$ & $10(12.0)$ \\
\hline $\begin{array}{l}\text { Prospective participation in cancer clinical trials } \\
\text { Yes }\end{array}$ & 55 (66.3) \\
\hline $\begin{array}{l}\text { Have you ever received any training course on how to } \\
\text { conduct clinical research? } \\
\text { Yes }\end{array}$ & 37 (44.6) \\
\hline $\begin{array}{l}\text { Do you consider it is necessary for physicians to conduct } \\
\text { clinical research? } \\
\text { Yes }\end{array}$ & $76(91.6)$ \\
\hline
\end{tabular}


Table 3 Knowledge of Medical Residents Toward Cancer Clinical Trials

\begin{tabular}{|l|l|}
\hline Item & N (\%) \\
\hline $\begin{array}{l}\text { Have you ever helped in writing a cancer clinical trial } \\
\text { protocol? }\end{array}$ & 2 I (25.3) \\
Yes & \\
\hline $\begin{array}{l}\text { Have you submitted for publication a manuscript on } \\
\text { cancer clinical trials? } \\
\text { Yes }\end{array}$ & $24(28.9)$ \\
\hline Do you know the purpose of the Declaration of Helsinki? \\
\hline $\begin{array}{l}\text { I know very well } \\
\text { I know to some extent }\end{array}$ & I (I.2) \\
I do not know & $15(18.1)$ \\
\hline $\begin{array}{l}\text { According to the Declaration of Helsinki, the use of } \\
\text { placebo is acceptable under that no proven treatment } \\
\text { exists. } \\
\text { (Yes) }\end{array}$ & $62(74.7)$ \\
\hline \begin{tabular}{l} 
Informed consent from the participants should be obtained in: \\
\hline $\begin{array}{l}\text { All the clinical studies } \\
\text { Depends on the purpose of the clinical trial }\end{array}$
\end{tabular} & $56(67.5)$ \\
\hline $\begin{array}{l}\text { Do you know the meaning of clinical equipoise in } \\
\text { clinical trials? } \\
\text { (Yes) }\end{array}$ & $52(62.7 \%)$ \\
\hline $\begin{array}{l}\text { Using the new investigational drug to treat resistant } \\
\text { cancer without } \\
\text { considering patients well-being. } \\
\text { (Yes) }\end{array}$ & 37 (44.6) \\
\hline
\end{tabular}

Fifteen residents knew about the Declaration of Helsinki to some extent, and 67 of them did not know about it (Table 3). Most of the respondents answered that informed consent should be obtained for all clinical studies (Table 3). Fifty-two respondents were aware of the equipoise term clinical trials and general aspects related to it. (Table 3). A total number of 37 residents believed that they could use the new investigational drugs to treat resistant cancers without considering patients' well-being (Table 3). Finally, $84.3 \%$ of respondent believed that bias which might accompany clinical trials, limited resources and budget, and cultural beliefs are the main challenges that might be encountered by the clinical trial administration in Jordan (data not shown in tables)

\section{Discussion}

In this questionnaire-based study involving medical residents who had served the cancer patients during their residency training. Less than half of the respondents reported either previous or current participation in clinical trials in general. These findings were presumed by taking into consideration the relatively small number of clinical trials carried out in Jordan. ${ }^{18}$ Almost one third of the world's clinical trials are carried out outside the United States, but the contribution toward these trials from the Middle East and North Africa is considered significantly low representing less than $1 \%$ of the world's clinical trials. ${ }^{19}$ The current study showed that medical residents have a positive attitude toward future participation in clinical studies and thought that such studies could be highly beneficial for patients. Most participants believed that physicians should carry out clinical trials parallel to their regular work. Current findings were in agreement with results from other studies done in other developing countries. ${ }^{11,16,17,20}$ Although a large percentage of medical residents reported that they have taken training courses about clinical research at some point during their clinical training, only a small number of them showed basic knowledge about ethical standards related to patients' privacy and rights protection codes. More than two thirds of respondent residents showed adequate knowledge about informed consent importance and elements as well as when it is mandatory to be taken. Studies from Nepal and India reported that a large percentage of resident doctors and dental faculty were unaware of core ethical documents and codes related to clinical research such as the Declaration of Helsinki and the Nuremberg Code. ${ }^{21,22}$ Similar studies from the USA, Japan, Saudi Arabia, and India showed that physicians involved in cancer clinical trials had little knowledge or experience about clinical trials, yet they had high interest and positive attitude to learn more about the roles they could play in clinical trial research. ${ }^{11,16,20,23}$ These findings suggest the need to provide physicians, especially those planning to run future clinical trials, with the proper training involving the ethical challenges and other obstacles that they might encounter in the future.

During their residency training, medical residents should demonstrate strong knowledge and skills for effective treatments utilizing the best available medical resources and primary literature. Improving the resident's knowledge and attitudes toward clinical trials will certainly improve the health-care service provided to patients. Previous reports demonstrated that enhancing the understanding of clinical trials among primary physicians would help in relieving the patient's confusion and worries about participation in clinical trials. In addition, it will increase 
physicians' capacity for patients' referrals to clinical trials. ${ }^{20}$ Residents with both strong research and clinical experience are generally more recognized when applying for academic positions or highly competitive subspecialty fellowships. $^{24,26}$ Taken altogether, it is clear that early involvement of residents in clinical research will reflect positively on their clinical performance and career advancement. ${ }^{27}$

The majority of respondent residents attributed the challenges which might be encountered in the oncology clinical trials to many reasons, including lack of funding, proper infrastructure, cultural beliefs, and lack of patients' willingness to participate in clinical trials, and improper understanding of the regulations pertaining clinical trials practice in Jordan. Similar studies attributed the low number of conducted clinical trials in the developing countries to various reasons including the lack of financial and human capacity, ethical and regulatory system obstacles, lack of proper research environment, operational barriers, difficulty in recruiting participant patients in clinical trials, busy schedule for physicians, and lack of time availability to conduct and follow-up for clinical trials. ${ }^{28,30}$

The current study did have several strengths. To best of our knowledge, this study is the first to evaluate the medical residents' attitudes and knowledge toward cancer related clinical trials in Jordan. This study will be utilized in the future as a basis to draw the attention toward the need of better training for physicians, especially those in their early career regarding aspects and requirements of clinical trials research as per international standards.

The current study had some limitations including the fact that it was conducted in one research center in Jordan, and it did not involve other hospitals or centers where cancer patients are treated. Although the study data were collected three years ago, we believe that our findings are still relevant today as we have observed that clinical trials have not changed much over the past few years. Our next step includes incorporating more hospitals in Jordan to obtain a better understanding of the current situation in Jordan about physician knowledge and attitudes toward clinical research, aiming to maximize the patient's benefits.

\section{Conclusion}

The current study suggests that there is a strong need to improve the medical residents' knowledge towards the cancer clinical trials. Current findings indicate that it is necessary to obtain a better understanding of the ethical challenges and clinical research barriers which may be encountered by medical residents when they are involved in clinical trials. Obtaining an ideal research environment will create a high-quality level of clinical research, and more reliable evidence-based medicine recommendations for future cancer management.

\section{Abbreviations}

KAUH, King Abdullah University Hospital; JFDA, Jordan Food and Drug Administration; IRB, Institutional Review Board; ACGME, Accreditation Councils for Graduate Medical Education; NIH, National Institute of Health.

\section{Acknowledgments}

Work on this project was supported by grant \#5R25TW010026-02 from the Fogarty International Center of the US National Institutes of Health on behalf of the Research Ethics Program in Jordan. The funders had no role in the study design, data collection and analysis, and decision to publish.

\section{Disclosure}

The authors report no conflicts of interest in this work.

\section{References}

1. Zon R, Meropol NJ, Catalano RB, Schilsky RL. American society of clinical oncology statement on minimum standards and exemplary attributes of clinical trial sites. J Clin Oncol. 2008;26(15):2562-2567. doi:10.1200/JCO.2007.15.6398

2. Nardini C. The ethics of clinical trials. Ecancermedicalscience 2014;8:387.

3. Shanholtz C. Acute life-threatening toxicity of cancer treatment. Crit Care Clin. 2001;17(3):483-502. doi:10.1016/S0749-0704(05)70196-2

4. Samphao S, Eremin JM, Eremin O. Oncological emergencies: clinical importance and principles of management. Eur J Cancer Care (Engl). 2010;19(6):707-713. doi:10.1111/j.1365-2354.2009.01091.x

5. Ghose S, Radhakrishnan V, Bhattacharya S. Ethics of cancer care: beyond biology and medicine. Ecancermedicalscience. 2019;13:911. doi:10.3332/ecancer.2019.911

6. Wistuba II, Gelovani JG, Jacoby JJ, Davis SE, Herbst RS Methodological and practical challenges for personalized cancer therapies. Nat Rev Clin Oncol. 2011;8(3):135-141. doi:10.1038/ nrclinonc.2011.2

7. Nathan DG. Clinical research: perceptions, reality, and proposed solutions. National institutes of health director's panel on clinical research. JAMA. 1998;280(16):1427-1431. doi:10.1001/jama.280.16.1427

8. Common Program Requirements. Accreditation council for graduate medical education. Available from: https://www.acgme.org/What-We-Do/ Accreditation/Common-Program-Requirements. Accessed June 23, 2020.

9. Hamann KL, Fancher TL, Saint S, Henderson MC. Clinical research during internal medicine residency: a practical guide. Am $\mathrm{J} \mathrm{Med}$. 2006;119(3):277-283. doi:10.1016/j.amjmed.2005.12.001

10. Hernandez-Suarez D, San Martin MT, Colon-Marquez JA, JimenezVelazquez IZ, Lopez-Candales A. Continued need for clinical research in medical residency: from physician to clinical-translational researcher. $P$ R Health Sci J. 2017;36(1):44-46. 
11. Pawar DB, Gawde SR, Marathe PA. Awareness about medical research among resident doctors in a tertiary care hospital: A crosssectional survey. Perspect Clin Res. 2012;3(2):57-61. doi:10.4103/ 2229-3485.96446

12. Hayward RA, Taweel F. Data and the internal medicine houseofficer: alumni's views of the educational value of a residency program's research requirement. J Gen Intern Med. 1993;8(3):140-142. doi:10.1007/BF02599759

13. Smith M. Research in residency: do research curricula impact postresidency practice? Fam Med. 2005;37(5):322-327.

14. Jordan Food and Drug Administration Available from: https://www. jfda.jo. Accessed June 82020.

15. Annul Reports. Pharmaceutical Research Center-Jordan University of Science and Technology. Available from: http://www.just.edu.jo/ Centers/PharmaceuticalResearchCenter/Pages/Reports.aspx. Accessed June 8, 2020.

16. Sumi E, Murayama T, Yokode M. A survey of attitudes toward clinical research among physicians at kyoto university hospital. BMC Med Educ. 2009;9(1):75. doi:10.1186/1472-6920-9-75

17. Choudhury S, Pradhan R, Dubey L, et al. Knowledge and perception regarding clinical trials among doctors of government medical colleges: A questionnaire-based study. Perspect Clin Res. 2016;7(2):9499. doi:10.4103/2229-3485.179433

18. Clinical Trials. Available from: https:/clinicaltrials.gov/. Accessed June 102020.

19. Nair SC, Ibrahim H, Celentano DD. Clinical trials in the Middle East and North Africa (MENA) Region: grandstanding or grandeur? Contemp Clin Trials. 2013;36(2):704-710. doi:10.1016/j.cct.2013.05.009

20. Bylund CL, Weiss ES, Michaels M, et al. Primary care physicians' attitudes and beliefs about cancer clinical trials. Clin Trials. 2017;14 (5):518-525. doi:10.1177/1740774517717722

21. Adhikari S, Paudel K, Aro AR, Adhikari TB, Adhikari B, Mishra SR. Knowledge, attitude and practice of healthcare ethics among resident doctors and ward nurses from a resource poor setting, Nepal. BMC Med Ethics. 2016;17(1):68. doi:10.1186/s12910-016-0154-9
22. Mallela KK, Walia R, Tm CD, Das M, Sepolia S, Sethi P. Knowledge, attitudes and practice about research ethics among dental faculty in the north India. J Int Oral Health. 2015;7(Suppl 2):52-56.

23. Al-Tannir MA, Katan HM, Al-Badr AH, Al-Tannir MM, AbuShaheen AK. Knowledge, attitudes, practices and perceptions of clinicians towards conducting clinical trials in an academic tertiary care center. Saudi Med J. 2018;39(2):191-196. doi:10.15537/ smj.2018.2.21093

24. Potti A, Mariani P, Saeed M, Smego RA Jr. Residents as researchers: expectations, requirements, and productivity. Am J Med. 2003;115 (6):510-514. doi:10.1016/j.amjmed.2003.08.017

25. Souba WW, Tanabe KK, Gadd MA, Smith BL, Bushman MS. Attitudes and opinions toward surgical research. A survey of surgical residents and their chairpersons. Ann Surg. 1996;223(4):377-383. doi:10.1097/00000658-199604000-00006

26. Khan K, Levstik M. Ranking in Canadian gastroenterology residency match: what do residents and program directors want? Can J Gastroenterol. 2010;24(6):369-372. doi:10.1155/2010/537435

27. Seaburg LA, Wang AT, West CP, et al. Associations between resident physicians' publications and clinical performance during residency training. BMC Med Educ. 2016;16(1):22. doi:10.1186/s12909-0160543-2

28. Al-Dorzi HM, Naidu B, Khokhar S, White D, Arabi YM. Research experience, interest and perceived barriers of clinical staff working at the Intensive Care Department of a tertiary care academic hospital in Saudi Arabia. Middle East J Anaesthesiol. 2013;22(3):301-307.

29. Schlaff WD. Barriers to conducting clinical research in reproductive medicine around the world. Fertil Steril. 2011;96(4):801. doi:10.1016/j.fertnstert.2011.08.048

30. Seruga B, Sadikov A, Cazap EL, et al. Barriers and challenges to global clinical cancer research. Oncologist. 2014;19(1):61-67. doi:10.1634/theoncologist.2013-0290
International Journal of General Medicine

\section{Publish your work in this journal}

The International Journal of General Medicine is an international, peer-reviewed open-access journal that focuses on general and internal medicine, pathogenesis, epidemiology, diagnosis, monitoring and treatment protocols. The journal is characterized by the rapid reporting of reviews, original research and clinical studies

\section{Dovepress}

across all disease areas. The manuscript management system is completely online and includes a very quick and fair peer-review system, which is all easy to use. Visit http://www.dovepress.com/ testimonials.php to read real quotes from published authors. 